\title{
The Preconditioned SOR Iterative Method for Positive Definite Matrices
}

\author{
Yu-Qin Bai, Yan-Ping Xiao, and Wei-Yuan Ma \\ School of Computer Science and Information Engineering, Northwest University for Nationalities, Lanzhou, Gansu 730030, China \\ Correspondence should be addressed to Yu-Qin Bai; yqbai2006@163.com
}

Received 28 May 2013; Revised 20 August 2013; Accepted 20 August 2013

Academic Editor: Zhongxiao Jia

Copyright (C) 2013 Yu-Qin Bai et al. This is an open access article distributed under the Creative Commons Attribution License, which permits unrestricted use, distribution, and reproduction in any medium, provided the original work is properly cited.

We present several iterations for preconditioners introduced by Tarazaga and Cuellar (2009), and study the convergence of the method for solving a linear system whose coefficient matrix is positive definite matrices, and we also find that they complete very well with the SOR iteration, which is shown through numerical examples.

\section{Introduction}

For solving the large sparse linear system

$$
A x=b, \quad x, b \in R^{n},
$$

where $A \in R^{n \times n}$ is a square nonsingular positive definite matrix, an iteration method is often considered. For any splitting, $A=M-N$ with $\operatorname{det}(M) \neq 0$, the basic iterative method for system (1) is

$$
x^{k+1}=M^{-1} N x^{k}+M^{-1} b, \quad k=0,1, \ldots
$$

To improve the convergence rate of the basic iterative method, transform the original systems (1) into the preconditioner form

$$
P A x=P b,
$$

where $P$ is called the preconditioner or a preconditioning matrix. several preconditioned iterative methods have been proposed [1-6]. Since $P$ is nonsingular, (1) and (3) have the same solutions. We are considering here systems with a unique solution. It is well known that system (3) can be transformed by an iteration as follows:

$$
x^{k+1}=(I-P A) x^{k}+P b .
$$

This iteration is called the Richardson iteration for preconditioning system (3). In this paper, we consider the iteration methods by the following form:

$$
x^{k+1}=T x^{k}+f
$$

where $T$ represents the iteration matrix.

Lemma 1 (see $[7,8])$. For the iteration formula (5) to produce a sequence converging to $(I-B)^{-1} b$, for any starting point $x^{0}$, it is necessary and sufficient that the spectral radius of $B$ be less than one.

We will use the following notations. A matrix $A=\left(a_{i j}\right)$ is called a row diagonally dominant if

$$
\sum_{i \neq j}\left|a_{i j}\right|<\left|a_{i i}\right| \quad \text { for } i=1, \ldots, n,
$$

and column diagonally dominant if

$$
\sum_{i \neq j}\left|a_{i j}\right|<\left|a_{j j}\right| \quad \text { for } j=1, \ldots, n \text {. }
$$

The Frobenius inner product of $A$ and $B$ is defined by

$$
\langle A, B\rangle_{F}=\operatorname{trace}\left(A^{T} B\right),
$$

where trace $(A)$ denotes the trace of a matrix $A$, and $A^{T}$ stands for the transpose of $A$, and the spectral radius of a matrix $A$ 
is denoted by $\rho(A)$. Let $A$ be decomposed as $A=D-L-U$ in which $D$ is the diagonal of $A, L$ is the strict lower part of $A$, and $U$ is the strict upper part of $A$.

Using the Frobenius norm, the preconditioning matrix in [6] is given by

$$
P_{f}=\left(\begin{array}{cccc}
\frac{a_{11}}{\left\|a_{1}\right\|_{2}^{2}} & & & \\
& \frac{a_{22}}{\left\|a_{2}\right\|_{2}^{2}} & & \\
& & \ddots & \\
& & & \frac{a_{n n}}{\left\|a_{n}\right\|_{2}^{2}}
\end{array}\right) \text {, }
$$

where $a_{i}$ stands for the $i$ th row of the matrix $A$.

The second preconditioning matrix is $P_{1}=\alpha I$ and $\alpha$ is computed to minimize the infinity norm of the iteration matrix.

The small gap for a matrix $A$ is defined by

$$
\operatorname{sg}(A)=\min _{j=1, \ldots, n}\left(\left|a_{j j}\right|-\sum_{i \neq j}^{n}\left|a_{i j}\right|\right) .
$$

Obviously, $\operatorname{sg}(A)$ is positive for diagonally dominant matrices. we can also suppose the diagonal entries are positive, else, it is true by multiplying the corresponding rows with -1 .

Then, the preconditioner obtained by minimizing the infinity norm is given by

$$
\alpha=\frac{2}{\|A\|_{\infty}+\operatorname{sg}(A)} .
$$

We can easily find that the diagonal preconditioner is constant diagonal. Using the idea in [9], we can obtain the iterations associated with the preconditioners $P_{f}$ and $P_{\infty}$ as follows.

Solving the iteration

$$
x^{k+1}=\left(I-\omega P_{f} A\right) x^{k}+\omega P_{f} b,
$$

the matrix $A$ is decomposed by $A=D+L+U$ and $\omega P_{f} A$ is moved to the left-hand side, we obtain

$$
\left(I+\omega P_{f} L\right) x^{k+1}=\left(I-\omega P_{f}(D+U)\right) x^{k}+\omega P_{f} b .
$$

Because $I+\omega P_{f} L$ is inverse, we can easily get

$$
x^{k+1}=\left(I-\left(P_{f}^{-1}+\omega L\right)^{-1} A\right) x^{k}+\left(P_{f}^{-1}+\omega L\right)^{-1} \omega b
$$

or

$$
x^{k+1}=\left(I-\omega\left(I+\omega P_{f} L\right)^{-1} P_{f} A\right) x^{k}+\left(I+\omega P_{f} L\right)^{-1} \omega P_{f} b .
$$

This iteration is considered as sequential Frobenius norm iteration.

Similarly, we obtained and built the infinity norm iteration associated with $P_{\infty}$ as follows:

$$
x^{k+1}=\left(I-\left(P_{\infty}^{-1}+\omega L\right)^{-1} A\right) x^{k}+\left(P_{\infty}^{-1}+\omega L\right)^{-1} \omega b
$$

or

$$
x^{k+1}=\left(I-\omega\left(I+\omega P_{\infty} L\right)^{-1} P_{\infty} A\right) x^{k}+\left(I+\omega P_{\infty} L\right)^{-1} \omega P_{\infty} b .
$$

Now, we have set two preconditioned SOR iterative methods which use $P_{f}$ and $P_{\infty}$ as a preconditioner.

In this paper, first in Section 2, we discuss the convergence of the preconditioned SOR iterative method which uses $P_{f}$ and $P_{\infty}$ as a preconditioner. In Section 3, we provide numerical experiments to illustrate the theoretical results obtained in Section 2, and we find if we choose the set of parameters; then our method has smaller spectral radii of the iterative matrices than the SOR method, which is shown through numerical examples.

\section{Main Results}

\subsection{The Sequential Frobenius Norm Iteration}

Theorem 2. Suppose $1 \leq \omega<2$ and $A$ is positive definite; then the iteration

$$
x^{k+1}=\left(I-\left(P_{f}^{-1}+\omega L\right)^{-1} A\right) x^{k}+\left(P_{f}^{-1}+\omega L\right)^{-1} \omega b,
$$

converges for any starting point $x^{0}$.

Proof. By simple calculation, the iteration matrix can be written as

$$
\left(P_{f}^{-1}+\omega L\right)^{-1}\left(P_{f}^{-1}-(D+U)+(\omega-1) L\right),
$$

let $\lambda$ be corresponding eigenvalue; then

$$
\left(P_{f}^{-1}-(D+U)+(\omega-1) L\right) x=\lambda\left(P_{f}^{-1}+\omega L\right) x
$$

or

$$
\left(P_{f}^{-1}-A+L+(\omega-1) L\right) x=\lambda\left(P_{f}^{-1}+\omega L\right) x,
$$

we get that

$$
-A x=(\lambda-1)\left(P_{f}^{-1}+\omega L\right) x,
$$

then

$$
-x^{*} A x=(\lambda-1) x^{*}\left(P_{f}^{-1}+\omega L\right) x .
$$

The following proof is similar in [9], so we omit it; then the theorem is obtained.

\subsection{The Sequential Infinity Norm Iteration}

Theorem 3. Suppose $1 \leq \omega<2$ and $A$ is positive definite. If every diagonal entry of $A$ satisfies

$$
\|A\|_{\infty}+\operatorname{sg}(A)>\omega a_{i i},
$$

then the iteration

$$
x^{k+1}=\left(I-\left(P_{\infty}^{-1}+\omega L\right)^{-1} A\right) x^{k}+\left(P_{\infty}^{-1}+\omega L\right)^{-1} \omega b
$$

converges for any starting point $x^{0}$. 




(a)

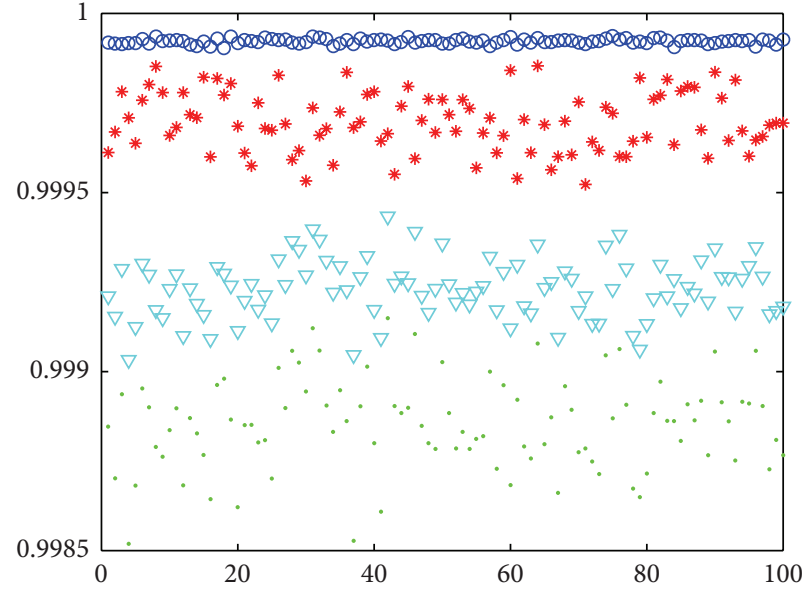

(b)



(c)

Figure 1: Spectral radius for random PSD matrices $A^{T} A+\operatorname{diag}(\operatorname{rand}(n, 1))$ of size $n=50(\mathrm{a}), A^{T} A+10 * \operatorname{diag}(\operatorname{rand}(n, 1))$ of $\operatorname{size} n=50(\mathrm{~b})$ and $A^{T} A+30 * \operatorname{diag}(\operatorname{rand}(n, 1))(\mathrm{c})$ of size $n=150$.

Proof. The proof of this theorem is similar to the previous one. We notice the diagonal entries of the matrix $2 P_{\infty}^{-1}-\omega D$ are

$$
\|A\|_{\infty}+\operatorname{sg}(A)-\omega a_{i i}
$$

by the assumption $\|A\|_{\infty}+\operatorname{sg}(A)>\omega a_{i i}$, so the diagonal entries of matrix $2 P_{\infty}^{-1}-\omega D$ are positive which completes the proof.

Now, we modify the infinity norm preconditioner for diagonally dominant matrices. Since the eigenvalues of a positive definite matrix $A$ lie in the interval $\left(0, \omega\|A\|_{\infty}+\epsilon\right)$ for arbitrarily small number $\epsilon$, the preconditioning matrix $P$ is defined by

$$
\widehat{P}_{\infty}=\frac{2}{\omega\|A\|_{\infty}+\epsilon},
$$

for any $\epsilon>0$. Especially, $\epsilon=0$ if $\rho(A)<\|A\|_{\infty}$.
Theorem 4. For any $\epsilon>0$, if $A$ is positive definite; then the iteration

$$
x^{k+1}=\left(I-\left(P_{\infty}^{-1}+\omega L\right)^{-1} A\right) x^{k}+\left(P_{\infty}^{-1}+\omega L\right)^{-1} \omega b,
$$

converges for any starting point $x^{0}$, where $1 \leq \omega<2$.

Proof. The proof is similar to Theorem 2, we notice the condition of

$$
2 P_{\infty}^{-1}-\omega D+\omega A>A
$$

or $2 P_{\infty}^{-1}-\omega D>0$, and the diagonal entries are

$$
2\left(\frac{\omega\|A\|_{\infty}+\epsilon}{2}\right)-\omega a_{i i}=\omega\|A\|_{\infty}+\epsilon-\omega a_{i i}>0 .
$$

Hence, we obtain this result.

Next, we will obtain a general result for previously positive definite preconditioners. 


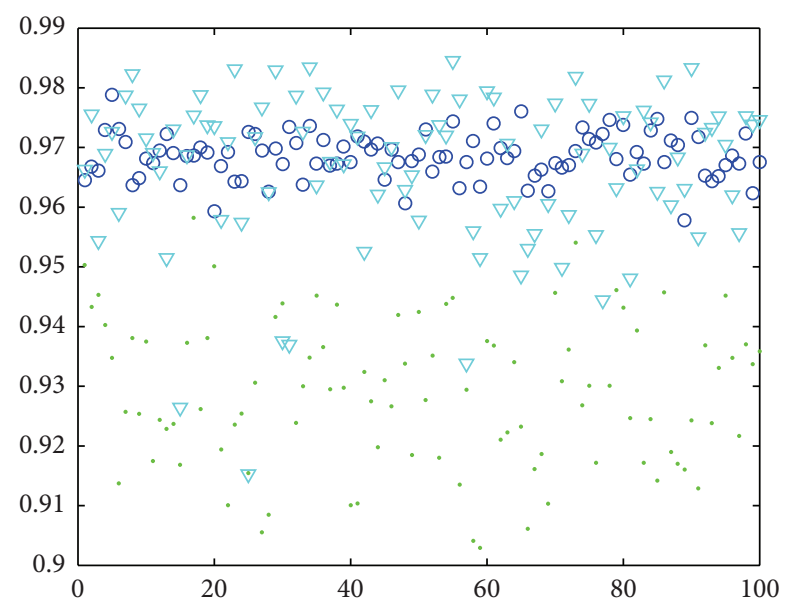

FIgURE 2: Spectral radius of random PSD matrices $A^{T} A+10 *$ $\operatorname{diag}(\operatorname{rand}(n, 1))$ with nearly half of the entries of $A$ negative and $n=50$.

Theorem 5. Suppose positive definite matrices of $A$ and $P$ satisfy

$$
2 P^{-1}-\omega D>0,
$$

where $D$ is the diagonal of $A$ and $1 \leq \omega<2$; then the iteration

$$
x^{k+1}=\left(I-\left(P^{-1}+\omega L\right)^{-1} A\right) x^{k}+\left(P^{-1}+\omega L\right)^{-1} \omega b,
$$

converges for any starting point $x^{0}$.

Remark 6. In Theorem 5, the matrix $P$ just satisfies the condition needed in the proof of Theorem 2; hence, this theorem is a general result for previous preconditioners.

\section{Numerical Experiments}

In this section, we provide numerical experiments to illustrate the theoretical results obtained in Section 2. All numerical experiments are carried out using MATLAB 7.1. The spectral radii of various iteration matrices are shown in Figures 1 and 2. For simplicity of comparison, suppose that all of $\omega=1.9$. Let $(o)$ denote the spectral radii of the SOR iteration matrices, let $(\cdot)$ denote the spectral radii of the Frobenius norm preconditioner iteration matrices, let $(*)$ denote the spectral radii of the infinity norm preconditioner, and let $(\nabla)$ denote the spectral radii of the infinity- $\epsilon$ preconditioner with $\epsilon=0.1$.

Remark 7. The previous numerical experiments indicate that the spectral radii of iterative matrices with three proposed preconditioners achieve significant improvement over the spectral radii of SOR iterative matrices.

Remark 8. As the proportion of negative to positive entries is increased, the spectral radius of random positive PSD matrices $A^{T} A+10 * \operatorname{diag}(\operatorname{rand}(n, 1))$ with half of the entries of $A$ negative with infinity norm preconditioner $(*)$ becomes larger than 1. But both of infinity norm preconditioners have faster convergence rates than SOR method.

Remark 9. Here, we maintain both of infinity norm iterations because there are cases when $P_{\infty}=\left(2 /\left(\|A\|_{\infty}+\operatorname{sg}(A)\right)\right)(*)$ works better than $\widehat{P}_{\infty}=\left(2 /\left(\omega\|A\|_{\infty}+\epsilon\right)\right)(\nabla)$.

\section{Acknowledgments}

The authors would like to thank the anonymous referees for their helpful comments and advice, which greatly improved the paper. The study was financially supported by National Natural Science Foundation (nos. 11161041, 71301111), Fundamental Research Funds for the Central Universities (31920130005).

\section{References}

[1] A. D. Gunawardena, S. K. Jain, and L. Snyder, "Modified iterative methods for consistent linear systems," Linear Algebra and Its Applications, vol. 154-156, pp. 123-143, 1991.

[2] T. Kohno, H. Kotakemori, H. Niki, and M. Usui, "Improving the modified Gauss-Seidel method for Z-matrices," Linear Algebra and Its Applications, vol. 267, pp. 113-123, 1997.

[3] A. Hadjidimos, D. Noutsos, and M. Tzoumas, "More on modifications and improvements of classical iterative schemes for M-matrices," Linear Algebra and Its Applications, vol. 364, pp. 253-279, 2003.

[4] W. Li and W. Sun, "Modified Gauss-Seidel type methods and Jacobi type methods for Z-matrices," Linear Algebra and Its Applications, vol. 317, no. 1-3, pp. 227-240, 2000.

[5] D. Noutsos and M. Tzoumas, "On optimal improvements of classical iterative schemes for Z-matrices," Journal of Computational and Applied Mathematics, vol. 188, no. 1, pp. 89-106, 2006.

[6] P. Tarazaga and D. Cuellar, "Preconditioners generated by minimizing norms," Computers \& Mathematics with Applications, vol. 57, no. 8, pp. 1305-1312, 2009.

[7] D. Kincaid and W. Cheney, Numerical Analysis: Mathematics of Scientific Computing, Brooks/Cole Publishing Co., Pacific Grove, Calif, USA, 2002.

[8] Y. Saad, Iterative Methods for Sparse Linear Systems, PWS Publishing Company, Boston, Mass, USA, 1996.

[9] R. L. Carden and P. Tarazaga, "Sequential iterations for two diagonal preconditioners," Computers \& Mathematics with Applications, vol. 58, no. 1, pp. 88-94, 2009. 


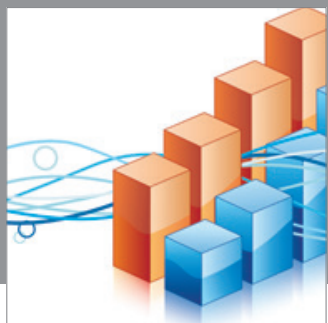

Advances in

Operations Research

mansans

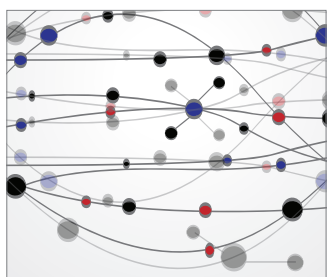

The Scientific World Journal
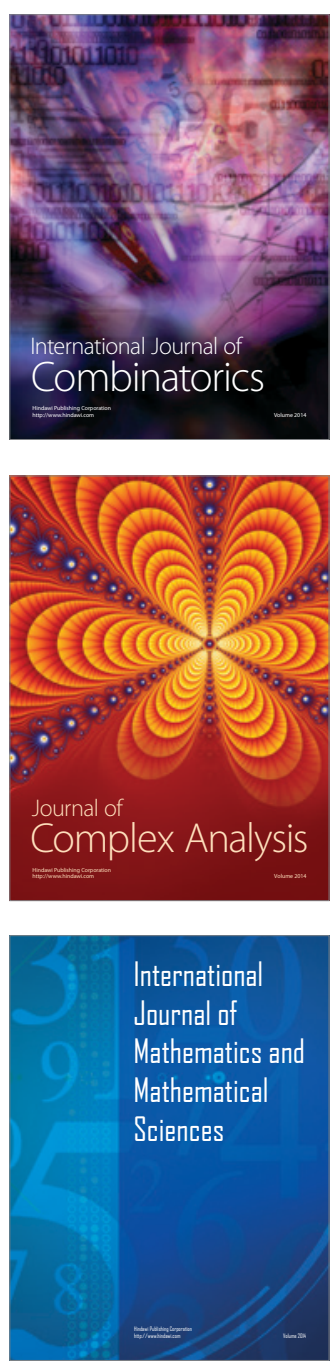


Submit your manuscripts at http://www.hindawi.com
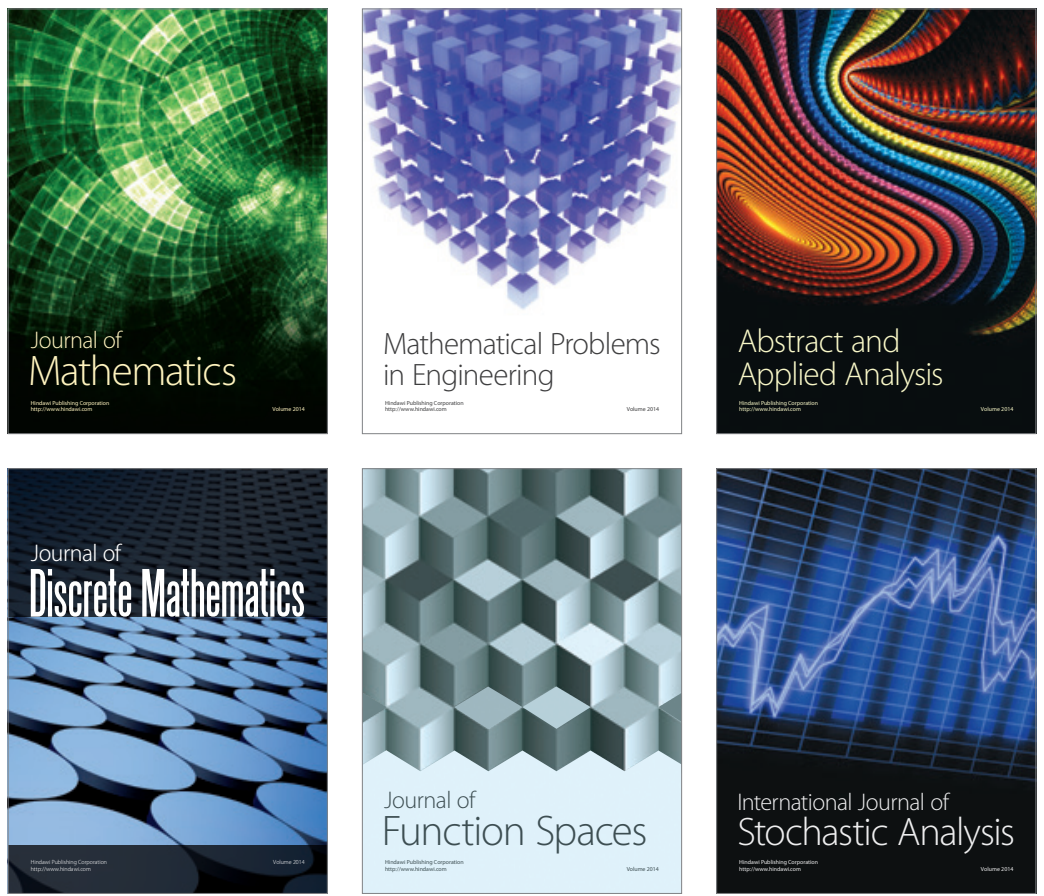

Journal of

Function Spaces

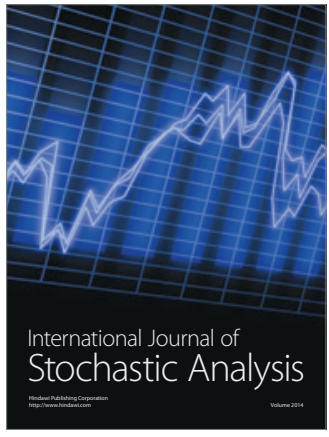

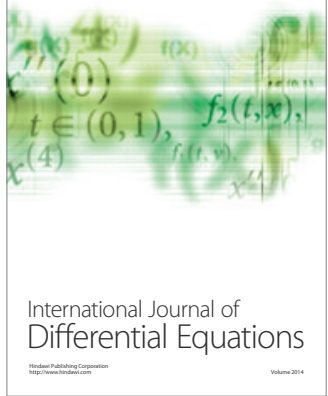
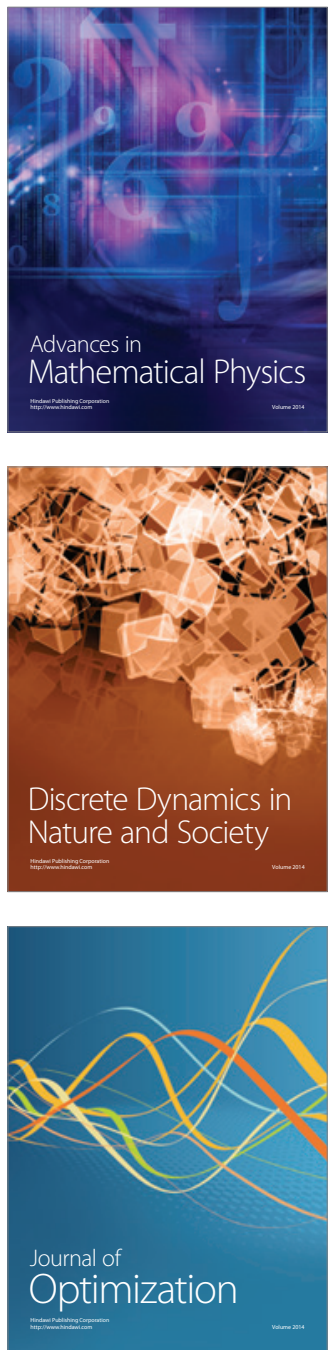